\title{
Sincronização entre um oscilador de fase e um forçamento externo
}

\author{
Synchronization between a phase oscillator and an external forcing \\ Ricardo Luiz Viana*1, Fabiane Fatima de Carvalho ${ }^{1}$ \\ ${ }^{1}$ Departamento de Física, Universidade Federal do Paraná, Curitiba, PR, Brasil
}

\begin{abstract}
Recebido em 11 de Outubro, 2016. Revisado em 09 de Janeiro, 2017. Aceito em 09 de Janeiro, 2017.
Vários sistemas de interesse físico e biológico podem ser descritos como osciladores auto-sustentados, que mantém seu comportamento oscilatório por meio de um aporte de energia. Do ponto de vista dinâmico, o comportamento de tais sistemas pode ser descrito por meio de um ciclo limite estável no espaço de fase, e o movimento sobre ele é parametrizado por uma fase com uma dada frequência natural. Neste trabalho investigamos o forçamento externo periódico no tempo deste tipo de sistema, destacando os fenômenos de sincronização de frequências, travamento de fase e sincronização generalizada. Um mapa unidimensional é obtido para estender o tratamento matemático geral, que supõe forçamento débil, para o caso de forçamento intenso.
\end{abstract}

Palavras-chave: osciladores, sincronização, fase, forçamento

Several systems of physical and biological interest can be described as self-sustained oscillators, which keep their oscillatory behavior using an external energy supply. From the dynamical point of view, the behavior of such systems can be described by means of a stable limit cycle in phase space, on which the motion can be parameterized by a phase with a given natural frequency. In this work we investigate the time-periodic external forcing acting on this kind of system, emphasizing frequency synchronization, phase locking and generalized synchronization. A one-dimensional mapping is obtained in order to extend the general mathematical treatment - which supposes weak forcing - for the case of strong forcing.

Keywords: oscillators, synchronization, phase, forcing

\section{Introdução}

É comum entre estudantes a ideia de que um sistema mecânico só pode manter oscilações estacionárias se houver um forçamento externo periódico. Esta noção é reforçada pelos tipos de osciladores mais estudados nos cursos básicos, como o sistema massamola e o circuito LC [1,2]. No entanto, há um grande número de sistemas de interesse físico e biológico que sustentam oscilações com um aporte externo constante de energia.

Em um trabalho anterior foram mostrados alguns exemplos simples desta categoria de sistemas, como o monjolo e o vaso de tântalo (sifonado), que são chamados osciladores de relaxação [3]. Estes, por

*Endereço de correspondência: viana@fisica.ufpr.br sua vez, fazem parte de uma categoria mais geral que chamaremos de osciladores auto-sustentados [4].

Devido à existência de uma oscilação estável e periódica, a dinâmica de osciladores auto-sustentados pode ser frequentemente reduzida a uma fase geométrica, cuja taxa de variação com o tempo é uma frequência característica. Estes osciladores de fase são sistemas que, embora muito simples matematicamente, exibem comportamentos dinâmicos ricos, especialmente quando são acoplados a um forçamento externo ou a outros osciladores similares [5].

O objetivo deste trabalho é investigar matematicamente o comportamento de um oscilador de fase, quando sujeito a um forçamento externo periódico no tempo. Como veremos, a riqueza dinâmica está ligada à não-linearidade do acoplamento entre o oscilador e o forçamento. Iremos descrever uma gama 
de fenômenos, como a ressonância simples e generalizada, bem como o comportamento quase-periódico. Serão usados dois enfoques: para forçamento débil, podemos fazer uma aproximação que destaca o comportamento nas vizinhanças de uma ressonância qualquer, estabelecendo de forma bastante geral as características da resposta do oscilador ao forçamento. Já para forçamento mais intenso, é necessário integrar as equações dos osciladores acoplados, o que é possível no caso particular de um forçamento periódico impulsivo, que reduz o sistema a uma equação a diferenças.

Este artigo está estruturado da seguinte forma: na Seção II nós discutimos alguns exemplos físicos e biológicos de osciladores auto-sustentandos, dando na Seção III uma descrição matemática para os mesmos, onde introduzimos o conceito de oscilador de fase, em especial o oscilador uniforme. Na Seção IV abordamos a sincronização de um oscilador uniforme sujeito a um forçamento externo periódico de pequena intensidade. A Seção V é dedicada à discussão do fenômeno de sincronização generalizada. O caso de forçamento de grande intensidade é discutido na Seção VI. A última Seção é destinada às Conclusões.

\section{Osciladores auto-sustentados}

Osciladores auto-sustentados podem manter suas oscilações a partir de um aporte constante de energia externa, que compensa a energia perdida por vários processos dissipativos que ocorrem no sistema. Há vários exemplos físicos e biológicos de osciladores auto-sustentados, cada qual com suas peculiaridades. Nesta seção vamos mostrar alguns destes exemplos, deixando uma discussão matemática mais elaborada para a próxima seção.

1. Sistemas do tipo adere-desliza ("stick-slip"): são sistemas onde, sob influência de uma força externa constante, há uma alternância entre deslizamento e aderência 6. Um exemplo típico é a vibração de uma corda de violino causada pelo movimento do arco. A corda adere ao arco na medida em que há um certo atrito estático entre as duas superfícies em contacto. No entanto a própria elasticidade da corda causa o aparecimento de uma força restauradora que rapidamente ultrapassa a força de atrito com o arco e provoca o deslizamento da corda. Após a liberação de energia a corda volta a aderir ao arco, e o processo continua, gerando uma oscilação periódica e auto-sustentada, pois o aporte de energia pelo movimento do arco é capaz de se contrapor aos efeitos dos atritos.

2. Relógio de pêndulo: A propriedade de isocronismo das pequenas oscilações de um pêndulo (isto é, o fato da frequência do mesmo ser independente da amplitude) já era conhecida por Galileo Galilei por volta de 1602 que, cerca de três décadas mais tarde, tentou construir com seu filho um relógio baseado nesta propriedade. Entretanto foi somente em 1656 que o holandês Christian Huyghens inventou o relógio de pêndulo, tendo patenteado o mesmo no ano seguinte [7]. A invenção posterior do mecanismo de escape por âncora permitiu restringir as amplitudes das oscilações do pêndulo e aumentar ainda mais a precisão do relógio [8]. Como o movimento do pêndulo sofre a ação de vários fatores que dissipam energia, para que o relógio possa funcionar é necessário um aporte de energia, como a queda lenta de um objeto pesado ou a liberação de energia por uma mola helicoidal. De fato, é o mecanismo de escape por âncora que fornece ao pêndulo os impulsos necessários ao seu movimento em cada oscilação, sendo identificável pelo seu característico som de "tic-tac". Desta forma, as oscilações do pêndulo são auto-sustentadas, pois o aporte de energia é constante e capaz de contrabalançar as diversas fontes de dissipação de energia.

3. Metrônomos: Muito usados na educação musical, são dispositivos que produzem ritmos com uma frequência bem-definida e regulável $[9]$. O tipo mais tradicional de metrônomo, inventado no início do século XIX, baseia-se num mecanismo similar ao relógio de pêndulo, mas neste caso utiliza um pêndulo invertido, com uma massa cuja posição é ajustável ao longo de uma haste metálica. Alterandose a posição da massa é possível variar a frequência com que o metrônomo indica o ritmo. Há ainda uma segunda massa fixa no outro lado do ponto de suspensão do pêndulo, usualmente oculta. A fonte de energia é um mecanismo de corda, baseado na liberação de energia por uma mola.

4. Circuito com lâmpada de Neônio: Num circuito elétrico com uma lâmpada de Neônio, esta só se acende se a diferença de potencial for superior a um certo limiar 10]. Ligando uma fonte de tensão DC a este circuito (aporte constante de energia), inicialmente o capacitor é carregado. Enquanto a tensão não for igual ao limiar, a lâmpada estará apagada. Quando a diferença de potencial atingir o limiar, a lâmpada acende e descarrega rapidamente 
o capacitor. Como resultado, a tensão diminui apagando a lâmpada e reiniciando o processo. Assim a lâmpada pisca a intervalos de tempo regulares [3].

5. Disparos de um neurônio Os neurônios são células nervosas que disparam pulsos elétricos quando excitados externamente por uma corrente elétrica constante 11. Os detalhes da biofísica que está por trás deste processo são complexos e não podem ser explicados detalhadamente em poucas palavras, mas a ideia básica é que existe uma diferença de potencial entre os lados externo e interno da membrana neuronal [12]. A evolução temporal desta diferença de potencial é afetada pelas concentrações de íons (principalmente sódio e potássio) dentro e fora do neurônio 13. Devido à existência de canais na membrana que permitem a passagem seletiva de íons, quando a diferença de potencial na membrana é superior a um certo limiar, então há o disparo de um pulso elétrico, e a consequente despolarização da membrana [14]. A emissão destes disparos pode ou não ser periódica e depende da existência de um aporte de energia representado pela corrente elétrica injetada. Na Figura 1 mostramos um exemplo de disparos para um neurônio descrito matematicamente pelas equações de Hindmarsh-Rose [15].

Algumas das características comuns a estes exemplos, e que podem ser tomados como representativos dos osciladores auto-sustentados são [5]

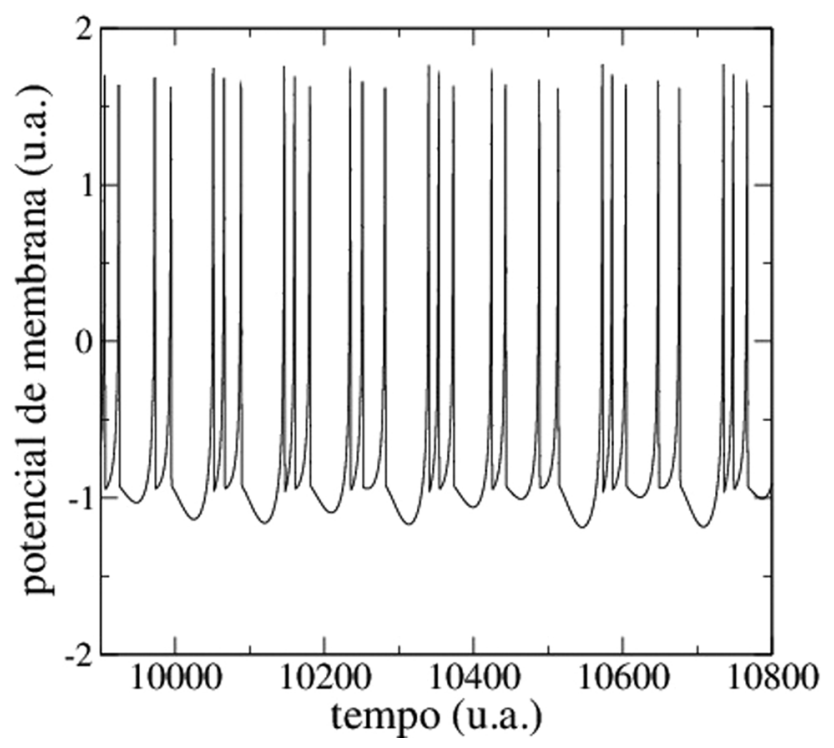

Figura 1: Evolução temporal do potencial de membrana $x(t)$ de um neurônio descrito pelas equações de HindmarshRose: $\dot{x}=y-x^{3}+3 x^{2}-z+I, \dot{y}=1-5 x^{2}-y, \dot{z}=$ $0,008[4(x+1,6)]-z$, com a corrente externa constante $I=3,25$ [15.
- os sistemas são ativos, contendo uma fonte interna de energia que é transformada em movimento oscilatório;

- os sistemas são isolados, pois o oscilador continua a gerar o mesmo ritmo até que a fonte de energia se esgote;

- o tipo de oscilação é determinado pelos parâmetros do sistema, e não pelas suas condições iniciais;

- as oscilações são estáveis contra pequenas perturbações, ou seja, após perturbadas elas retornam à sua forma inicial.

\section{Descrição por meio de osciladores de fase}

Em todos os exemplos acima, é possível (embora com graus de complexidade diferentes) fazer um modelamento matemático do oscilador. Supondo, por simplicidade, que haja apenas duas variáveis dinâmicas de interesse, que vamos chamar de $x$ e $y$, o estado do sistema num tempo $t$ poderá ser representado no plano de fase por um ponto de coordenadas $(x(t), y(t))$, e a evolução temporal do sistema por uma trajetória neste plano de fase. No caso do pêndulo, por exemplo, $x$ pode significar o ângulo entre o fio (ou uma haste muito leve) e a vertical, e $y$ a respectiva velocidade angular. Já no exemplo do circuito com lâmpada de neônio, $x$ seria a carga nas placas do capacitor e $y$ a corrente que passa pelo circuito.

Sob condições bastante gerais, existirá uma trajetória fechada e atrativa chamada ciclo limite neste plano de fase, representando o comportamento estacionário das oscilações auto-sustentadas. Mais precisamente, o ciclo limite é uma trajetória periódica pois, após um certo período $T$, os valores das variáveis retornam aos seus valores iniciais, ou seja: $(x(t+$ $T), y(t+T))=(x(t), y(t))$ [16. Um ciclo limite atrativo significa que as trajetórias cujas condições iniciais estejam em sua proximidade (dentro ou fora) convergem para o ciclo com o passar do tempo [Fig. 2].

Quando o sistema dinâmico atinge assintoticamente o estado estacionário, a sua trajetória coincide com o ciclo-limite. Sendo o ciclo-limite uma curva fechada no plano de fase, podemos usar coordenadas polares (em relação a um centro de rotação) para descrever a posição de um ponto $(r, \theta)$, tal que $x=r \cos \theta$ e $y=r \sin \theta$ [cf. Fig. 2]. O ângulo $\theta=\arctan (y / x)$ é denominado fase geométrica, tal 


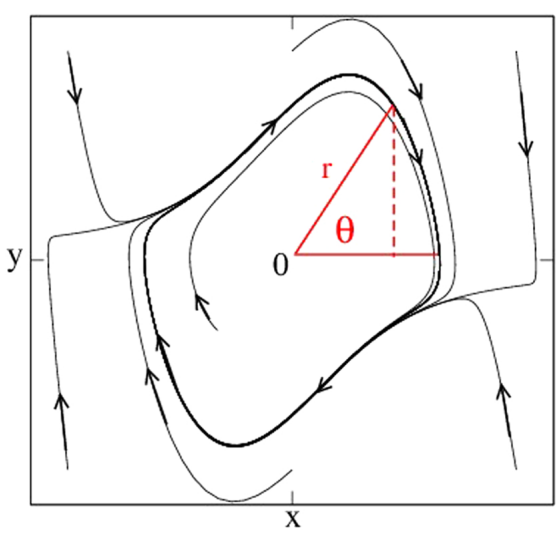

Figura 2: Um ciclo limite atrativo no plano de fase, e o significado geométrico da fase associada ao movimento.

que após um período completo de oscilação a fase ganha um acréscimo de $2 \pi$ radianos.

Em geral, como mostrado na Fig. 2, a fase varia com o tempo a uma taxa variável, dependendo da forma do ciclo-limite. Se este, no entanto, for um círculo, temos o chamado oscilador de fase uniforme, tal que a fase varia com o tempo com uma frequência constante $\omega_{0}=2 \pi / T$ ( $T$ é chamado período da oscilação)

$$
\frac{d \theta}{d t}=\omega_{0},
$$

e que pode ser imediatamente integrada fornecendo

$$
\theta(t)=\theta(0)+\omega_{0} t
$$

onde $\theta(0)$ é a fase inicial (em $t=0)$. Se $\omega_{0}>0$ a rotação da fase é convencionalmente no sentido anti-horário, enquanto se $\omega_{0}<0$ a rotação se dá no sentido horário. Ao longo de um ciclo completo de oscilação a fase cresce de $2 \pi$ em relação ao seu valor anterior. Podemos, assim, considerar o valor "cheio" da fase, que é ilimitado, ou então o valor "reduzido" da fase, que varia apenas de zero a $2 \pi$. A relação entre estes dois valores é dada pela operação "módulo $2 \pi$ ", que fornece o resto da divisão de $\theta$ por $2 \pi: \theta_{\text {reduzido }}=\theta_{\text {cheio }},(\bmod 2 \pi)$.

\section{Oscilador de fase com forçamento externo periódico}

Em vários problemas de interesse físico e biológico desejamos conhecer a resposta de um oscilador a um forçamento externo periódico com uma dada frequência $\omega$. Podemos encarar o forçamento externo como um oscilador caracterizado pela fase $\psi(t)$ e uma frequência $\omega$, que age sobre o oscilador uniforme com fase $\theta(t)$ e frequência $\omega_{0}$ descrito na seção anterior

$$
\begin{aligned}
\frac{d \theta}{d t} & =\omega_{0}+\varepsilon G(\theta, \psi), \\
\frac{d \psi}{d t} & =\omega,
\end{aligned}
$$

onde $\varepsilon$ é uma constante, denominada intensidade do forçamento, e $G$ é uma função com período $2 \pi$ tanto em $\theta$ como em $\psi$. Inicialmente vamos presumir que $\varepsilon$ seja uma quantidade muito pequena, de modo que o forçamento é débil.

Subtraindo a equação (4) da equação (3) obtemos

$$
\frac{d \varphi}{d t}=\Delta \omega+\varepsilon G(\theta, \psi)
$$

onde definimos a diferença de fase entre o oscilador e seu forçamento,

$$
\varphi=\theta-\psi
$$

e o descasamento entre as respectivas frequências

$$
\Delta \omega=\omega-\omega_{0}
$$

\subsection{Sincronização de frequências e travamento de fases}

Inicialmente voltamos nossa atenção ao caso em que estamos próximos à condição de ressonância, ou seja, $\omega \approx \omega_{0}$, para a qual o descasamento de frequências é pequeno. Neste pode-se mostrar (vide Apêndice) que, fazendo uma média sobre termos que oscilam rapidamente no tempo, o termo de acoplamento na equação (5) entre o oscilador e seu forçamento depende apenas da diferença de fase, e pode ser escrito como $g(\theta, \psi)=g(\theta-\psi)=g(\varphi)$. Uma função simples com período $2 \pi$ é $g(\varphi)=\sin \varphi$, com a qual a equação (5) torna-se

$$
\frac{d \varphi}{d t}=-\Delta \omega+\varepsilon \sin \varphi
$$

conhecida na literatura sobre circuitos eletrônicos como equação de Adler 17.

Muito embora possamos resolver analiticamente a equação acima, o resultado não nos ajuda muito a visualizar a dinâmica do problema. É melhor (e frequentemente mais fácil) investigar alguns de seus aspectos qualitativos mais importantes, a partir da análise no plano de fase $\dot{\varphi}$ versus $\varphi$ [Fig. 3(a)]. Por exemplo, o ponto de equilíbrio $\varphi^{*}$ é definido pela 
condição $d \varphi^{*} / d t=0$ que, uma vez resolvida, leva à solução

$$
\sin \varphi^{*}=\frac{\Delta \omega}{\varepsilon} .
$$

Como $\left|\sin \varphi^{*}\right| \leq 1$ resulta que, para que existam um ou mais pontos de equilíbrio, é necessário que, sendo $\varepsilon>0$, tenhamos

$$
|\Delta \omega| \leq \varepsilon .
$$

Supondo, sem perda de generalidade, que $\omega_{0}>$ $\omega$ (descasamento de frequências negativo) então a equação de Adler (21) torna-se

$$
\frac{d \varphi}{d t}=|\Delta \omega|+\varepsilon \sin \varphi .
$$

Aparecem aqui três situações possíveis: a primeira é quando $\varepsilon>|\Delta \omega|$, de sorte que a equação (9) terá duas soluções no intervalo $[\pi, 2 \pi)$ :

$$
\begin{aligned}
\varphi_{1}^{*} & =\arcsin \left(-\frac{|\Delta \omega|}{\varepsilon}\right), \\
\varphi_{2}^{*} & =\arcsin \left(-\frac{|\Delta \omega|}{\varepsilon}\right)+\frac{\pi}{2},
\end{aligned}
$$

sendo que a primeira solução é estável, e a segunda, instável, como podemos inferir da Fig. 3(a). Considerando apenas a solução de equilíbrio estável, isso significa que $\theta=\psi+\varphi_{1}^{*}$, ou seja, a diferença de fase é constante, o que é chamado travamento de fases ("phase-locking"). Resolvendo a equação (4) temos $\psi(t)=\omega t$, de modo que $\theta(t)=\omega t+$ const. Portanto, a frequência do oscilador forçado será

$$
\Omega=\frac{d \theta}{d t}=\omega,
$$

de modo que o oscilador responde à mesma frequência do forçamento. Esta sincronização de frequências também recebe o nome de "entrainment" (um termo sem uma tradução adequada em português) [16].

Há vários exemplos interessantes de sincronização de frequências entre um oscilador de fase (autosustentado) e um forçamento externo periódico. É possível sincronizar relógios analógicos por meio de sinais de rádio: um pulso eletromagnético gerado por um relógio central extremamente preciso (usualmente um relógio atômico) é usado para fazer pequenos ajustes em outros relógios de modo a sincronizá-los com esta central [18].

Outra aplicação consiste no ajuste dos chamados ritmos circadianos, que é o nome genérico dado a vários fenômenos em seres vivos com periodicidade aproximada de 24 horas [19]. O forçamento, nestes casos, é proveniente do ciclo dia-noite, regulado pela rotação da Terra e pela posição do Sol. Assim, mesmo que o ritmo circadiano tenha um período um pouco diferente, ele será sincronizado com o ciclo dianoite. Para dessincronizar estes ritmos circadianos é necessário viajar à regiões em altas latitudes ou então explorar cavernas, por exemplo 20.

Os ritmos circadianos permitem, ainda, exemplificar o efeito do travamento de fase. Há uma diferença de fase entre o ritmo circadiano e o ritmo inerente ao ciclo dia-noite, e que varia de pessoa para pessoa: há os madrugadores e os dorminhocos, para os quais o dia "começa mais cedo" ou "mais tarde", respectivamente. Viagens aéreas onde a longitude geográfica varia bastante (como cruzar o Oceano Atlântico ou Pacífico) levam a perturbações desta diferença de fase, ocasionando o chamado "jet-lag", que é o desconforto característico enquanto o relógio biológico (o ritmo circadiano) não ajusta a sua fase ao ciclo dia-noite [5]. Este ajuste também varia conforme o
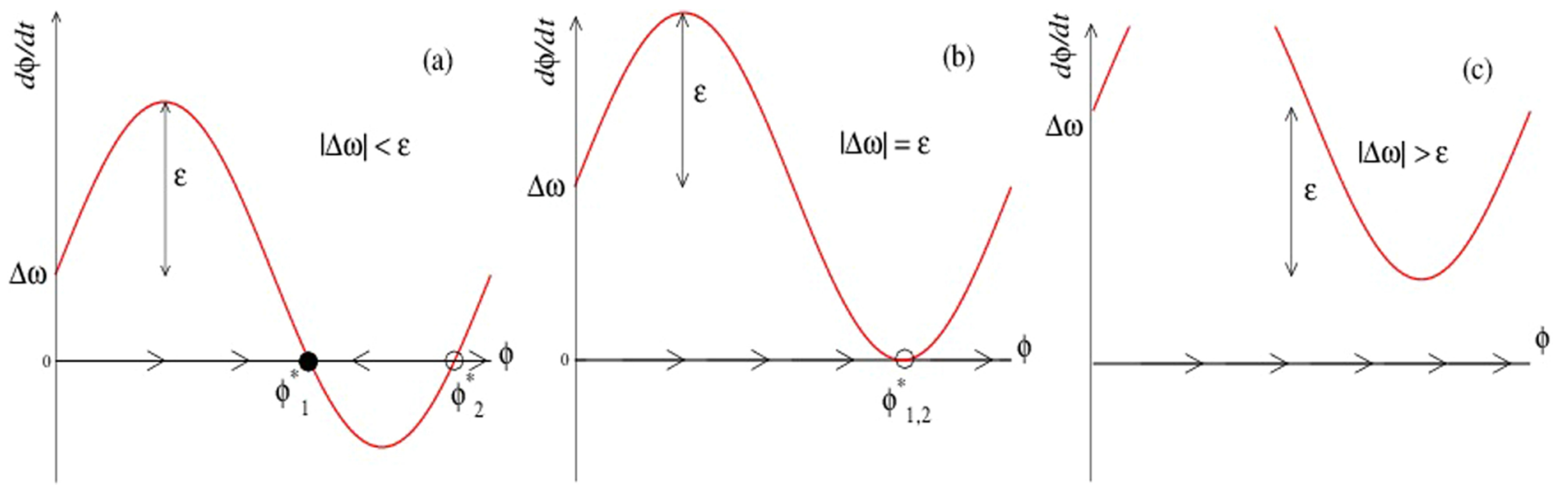

Figura 3: Plano de fase para os casos em que o descasamento de frequências é menor (a), igual (b) e maior (c) do que a intensidade do forçamento. 
indivíduo, e pode durar até vários dias em alguns casos.

\subsection{Batimentos e quase-periodicidade}

O caso $\varepsilon=|\Delta \omega|$ é tal que há apenas um ponto de equilíbrio $\varphi_{1}^{*}=\varphi_{2}^{*}=3 \pi / 2$, e que é semi-estável, ou seja, é estável pela esquerda e instável pela direita [Fig. 3(b)]. O comportamento é diferente, porém, no caso $\varepsilon<|\Delta \omega|$, que viola a condição (10), de modo que não há ponto de equilíbrio. Portanto não haverá sincronização do oscilador com o forçamento nem travamento de fases. De fato, derivando a equação (6) em relação ao tempo e usando as equações (4) e (14) temos

$$
\frac{d \varphi}{d t}=\Omega-\omega=-\delta \Omega
$$

onde $\delta \Omega \equiv \omega-\Omega$ é chamada frequência de batimento.

No caso anterior, onde havia sincronização de frequências, tínhamos $\delta \Omega=0$. Já no presente caso a equação de Adler (11) indica que o sistema torna-se um oscilador não-uniforme, pois sua frequência não é mais constante, dependendo agora da diferença de fase. O período do batimento $T_{\varphi}=2 \pi /|\delta \Omega|$ pode ser achado integrando a equação (15) ao longo de um ciclo completo, onde há um aumento de fase de $2 \pi$ :

$$
T_{\varphi}=\left|\int_{0}^{2 \pi} \frac{d \varphi}{\Delta \omega-\varepsilon \sin \varphi}\right|=\frac{2 \pi}{\sqrt{(\Delta \omega)^{2}-\varepsilon^{2}}} .
$$

Na ausência de forçamento $(\varepsilon=0)$, o período do batimento reduz-se a $2 \pi / \Delta \omega$, que é o período associado ao descasamento de frequência. $\mathrm{O}$ valor de $T_{\varphi}$ aumenta lentamente com $\varepsilon$ para pequenas intensidades do forçamento, e depois rapidamente à medida em que $\varepsilon$ aproxima-se do seu valor limite $\Delta \omega$ (para valores ainda maiores há sincronização, como vimos) [Fig. 4(a)]. O motivo é que, no caso em que $|\Delta \omega|$ é apenas ligeiramente maior que $\varepsilon$, formase um "gargalo" no plano de fase próximo ao valor $3 \pi / 2$ (que é o "fantasma" do ponto de equilíbrio para $\varepsilon=|\Delta \omega|)$. A diferença de fase aproxima-se lentamente deste gargalo e lá permanece durante um certo período, que tende a infinito na medida em que tendemos para $\varepsilon=|\Delta \omega|$. Na linguagem da teoria dos sistemas dinâmicos, dizemos que já ocorreu uma bifurcação tangente neste ponto, tal que os pontos de equilíbrio (um estável e outro instável) aproximam-se mutuamente e desaparecem após ultrapassado o ponto de bifurcação [21].

O comportamento para $\varepsilon$ na vizinhança do ponto de bifurcação pode ser obtido aproximando-se a raiz quadrada na equação (16) como

$$
\begin{aligned}
& \sqrt{(\Delta \omega)^{2}-\varepsilon^{2}}=\sqrt{\Delta \omega-\varepsilon} \sqrt{\Delta \omega+\varepsilon} \\
& \approx \sqrt{2 \Delta \omega} \sqrt{\Delta \omega-\varepsilon}, \quad(\varepsilon \lesssim|\Delta \omega|),
\end{aligned}
$$

que, substituído na equação (16), leva à seguinte expressão aproximada para a frequência de batimento nas vizinhanças do gargalo 21

$$
|\delta \Omega|=\sqrt{2 \Delta \omega}(\Delta \omega-\varepsilon)^{1 / 2}, \quad(\varepsilon \lesssim|\Delta \omega|) .
$$

Na Fig. 4(b) mostramos o gráfico da frequência de batimento em função do descasamento das frequências.
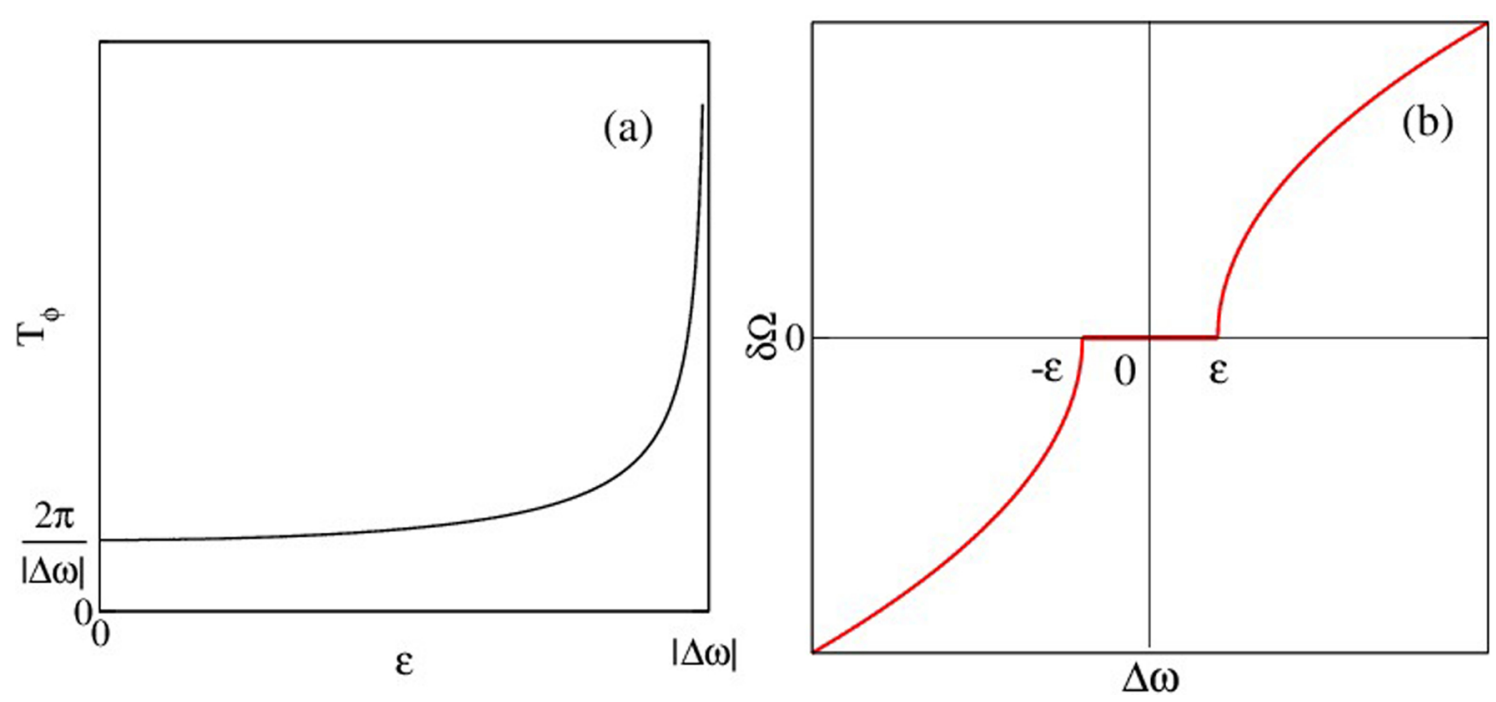

Figura 4: (a) Período do batimento em função da intensidade do forçamento; (b) Frequência de batimento em função do descasamento de frequências. 
Na região onde $-\varepsilon \leq \Delta \omega \leq \varepsilon$ há sincronização, portanto não há batimento. Fora deste intervalo, como vimos, a frequência de batimento aumenta ou diminui com a raiz quadrada da diferença entre $\Delta \omega$ e $\varepsilon$. Apesar disto $\varepsilon$ é suposto sempre pequeno (forçamento débil) neste tratamento.

Outra forma de apreciar esta diferença de comportamento é fazendo o gráfico da intensidade do forçamento versus o descasamento de frequência. Nele representamos a condição de sincronização (10) pela região limitada internamente pela função $\varepsilon=|\Delta \omega|$ [Fig. 5(a)]. Para pontos fora desta região não há sincronização, e sim o batimento descrito por (16). Nas variáveis $\varepsilon$ em função de $\omega$ este gráfico é chamado de plano de parâmetros do forçamento, e nele a região de sincronização (também conhecida como "língua de Arnold" 22]) é deslocada para o ponto de ressonância exata $\omega=\omega_{0}$ [Fig. [5(b)].

A existência desta língua de Arnold ajuda-nos a entender melhor o próprio conceito de ressonância, entendida como a igualdade exata entre as frequências do oscilador $\omega_{0}$ e do forçamento $\omega$. Se não há forçamento, ou se este é infinitamente pequeno, então a sincronização entre estas frequências só ocorre no ponto de ressonância. Entretanto, na medida em que a intensidade do forçamento cresce, a sincronização ocorre para valores ligeiramente diferentes de $\omega$ e $\omega_{0}$, desde que o módulo desta diferença não exceda $\varepsilon$. Se pensarmos que a frequência do forçamento pode flutuar ligeiramente em relação ao seu valor médio $\omega$, então a sincronização é um fenômeno "robusto", no sentido em que persiste mesmo ligeiramente fora da condição de ressonância exata.

\section{Sincronização generalizada}

A ressonância pode ocorrer também entre a frequência da força externa e um múltiplo ou sub-múltiplo da frequência natural do oscilador. De maneira geral, a condição de ressonância pode ser colocada na forma

$$
\omega=\frac{m}{n} \omega_{0},
$$

onde $m$ e $n$ são dois números inteiros, primos entre si. O caso abordado na seção anterior corresponde à escolha $m=n=1$. Para analisar esta situação usando o mesmo procedimento, redefinimos a diferença de fase como

$$
\varphi=m \theta-n \psi,
$$

e o descasamento entre as respectivas frequências como

$$
\Delta \omega=n \omega-m \omega_{0} .
$$

Se estamos próximos à ressonância para a qual $n \omega \approx m \omega_{0}$, nós também podemos considerar apenas o termo ressonante, fazendo uma média sobre o tempo, e que também dependerá apenas da diferença de fase: $\hat{g}(m \theta-n \psi)=\hat{g}(\varphi)$ (vide Apêndice). Multiplicando a equação (3) por $m$, a equação (4) por $n$, e subtraindo membro-a-membro os resultados, obtemos

$$
\frac{d \varphi}{d t}=-\Delta \omega+\varepsilon m \hat{g}(\varphi),
$$

a qual, pela escolha $\hat{g}(\varphi)=\sin \varphi$, também recai na equação de Adler. Vemos, assim, que para as sincronizações generalizadas repete-se o mesmo padrão que vimos na seção anterior: para cada ressonância onde
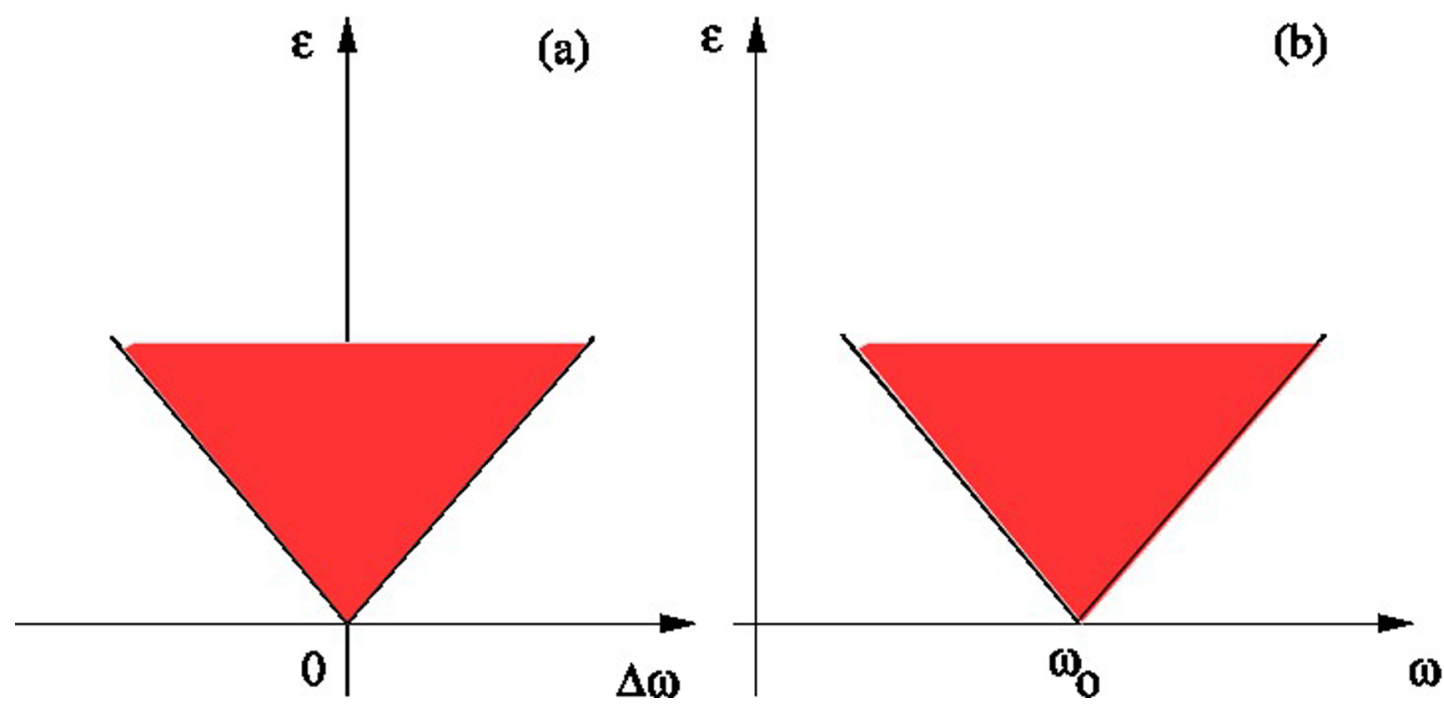

Figura 5: Região de sincronização nos planos (a) $\varepsilon$ versus $\Delta \omega$; (b) $\varepsilon$ versus $\omega$. 
$\omega / \omega_{0}=m / n$ haverá, para intensidade de forçamento diferente de zero, um ponto de equilíbrio estável $\varphi^{*}$ para a equação (21), tal que a equação $(19)$ fornece

$$
\theta(t)=\varphi^{*}+\frac{n}{m} \omega t
$$

correspondente a uma frequência do oscilador perturbado igual a

$$
\Omega=\frac{d \theta}{d t}=\frac{n}{m} \omega
$$

assim como a uma região de sincronização no plano $\varepsilon$ versus $\omega$ na forma de uma língua de Arnold ancorada em $\omega=m \omega_{0} / n$.

Por exemplo, sejam $m=1$ e $n=2$, tais que $\omega=\omega_{0} / 2$. Para $\varepsilon \neq 0$ haverá um platô de sincronização para $\Omega / \omega=2$, correspondendo a uma língua de Arnold $2: 1$ ancorada em $\omega=\omega_{0} / 2$ [Fig. 6]. É importante observar que, para uma mesma intensidade de forçamento, as larguras das línguas de Arnold $n: m$ serão, em geral, diferentes. Além disso tipicamente a ressonância mais larga é a principal, onde $m=n=1$, ao passo que quanto maiores forem os inteiros $m$ e $n$ menor será, comparativamente, a largura dos intervalos de sincronização.

Para entender o que ocorre fora das regiões de sincronização generalizada [representadas pelas línguas de Arnold na figura 6(a)] podemos recorrer ao exemplo dado na seção anterior, correspondente à escolha $g(\varphi)=\sin \varphi$ (equação de Adler). Vimos que, fora da região de sincronização, o oscilador tem um comportamento não-uniforme. Do ponto de vista dinâmico, chamamos o movimento, neste caso, de quase-periódico, uma vez que é caracterizado por um número infinitamente grande de frequências 22 . Na prática, a fase do oscilador aproxima-se e afastase de forma irregular da fase do forçamento externo. Este comportamento também ocorre entre as línguas de Arnold no caso de ressonância generalizada.

\section{Forçamentos moderado e intenso}

A teoria desenvolvida anteriormente é baseada na hipótese que o forçamento é débil o suficiente para que, na vizinhança de uma ressonância, a diferença de fase correspondente varie de forma lenta com o

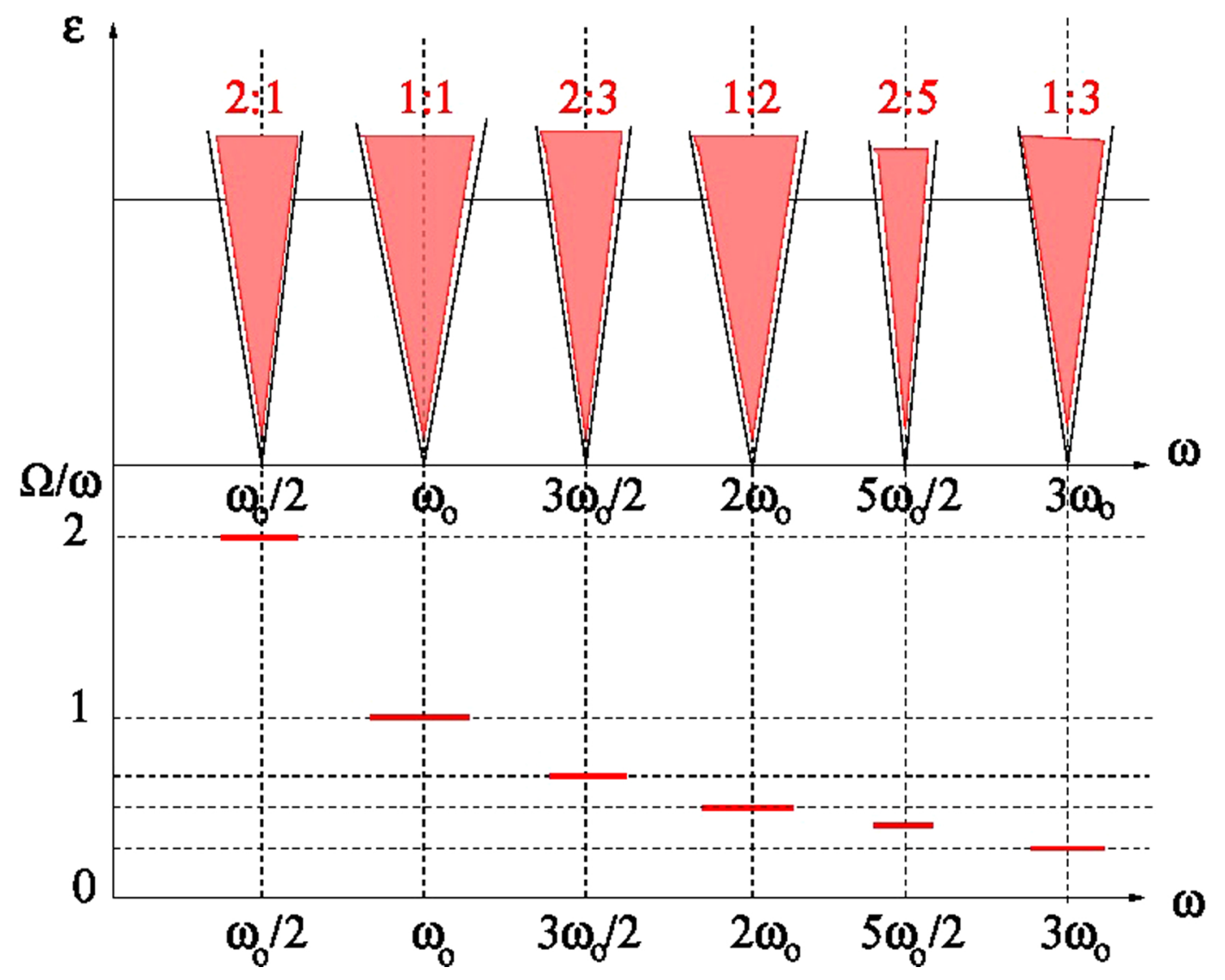

Figura 6: (a) Regiões de sincronização generalizada no plano $\varepsilon$ versus $\omega$. (b) Platôs de sincronização generalizada no diagrama $\Omega / \omega$ versus $\omega$. 
tempo. Se o forçamento for moderado, no entanto, esta hipótese já deixa de ser válida, de modo geral. Para entender o que ocorre neste caso, é necessário de alguma forma resolver as equações do movimento (3)-(4). Isto pode ser feito de maneira numérica para uma função não-linear arbitrária $G(\theta, \psi)$. Uma outra alternativa é supor uma forma conveniente para a função de forçamento que nos permita resolver parcialmente este conjunto de equações. Vamos, pois, supor a seguinte separação de variáveis

$$
G(\theta, \psi)=g(\theta) h(\psi)=g(\theta) h(\omega t),
$$

onde $g$ e $h$ são funções a serem especificadas. O tipo mais simples de forçamento é aquele que só depende do tempo, não exibindo dependência na fase, de modo que $g(\theta)=1$. Num tipo mais complicado de forçamento, a dependência na fase pode ser considerada uma modulação da dependência temporal, na forma dada pela equação (24).

Um caso particularmente interessante é o de uma sequência periódica de pulsos do tipo funções delta, na forma (o famoso "pente de Dirac")

$$
h(\omega t)=\sum_{m=-\infty}^{\infty} \delta(t-n T),
$$

onde $T=2 \pi / \omega$ é o período do forçamento externo [Fig. 7], de modo que a equação do oscilador forçado (3) é escrita como

$$
\frac{d \theta}{d t}=\omega_{0}+\varepsilon g(\theta) \sum_{m=-\infty}^{\infty} \delta(t-n T) .
$$

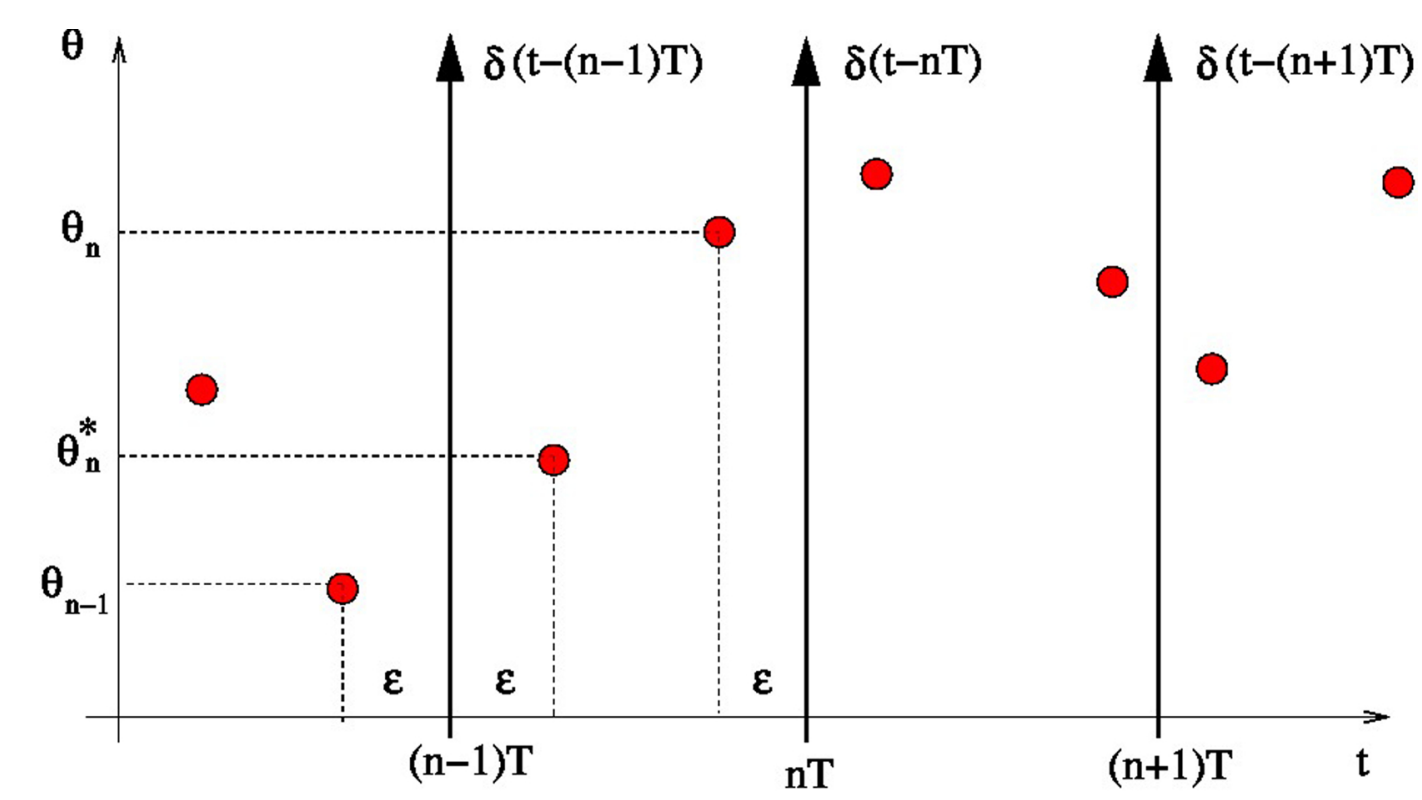

Figura 7: Definição das variáveis discretas para a obtenção do mapa do círculo.
O caráter impulsivo da perturbação permite-nos definir variáveis a tempo discreto. Definiremos $\theta_{n}$ como o valor da fase imediatamente antes da perturbação aplicada no tempo $t=n T$, ou seja

$$
\theta_{n}=\lim _{\epsilon \rightarrow 0} \theta(t=n T-\epsilon)
$$

e de maneira análoga para os instantes $t=(n-1) T$ e $t=(n+1) T$ [Fig. 7]. É útil, ainda, definir a variável discreta auxiliar $\theta_{n}^{*}$ como o valor da fase imediatamente depois da perturbação aplicada no tempo $t=(n-1) T$ :

$$
\theta_{n}^{*}=\lim _{\epsilon \rightarrow 0} \theta(t=(n-1) T+\epsilon) .
$$

Integrando a equação (26) no entorno da função delta em $t=(n-1) T$ e fazendo $\epsilon \rightarrow 0$ obtemos a seguinte relação entre as variáveis discretas:

$$
\theta_{n}^{*}-\theta_{n-1}=\varepsilon g\left(\theta_{n-1}\right) .
$$

No intervalo entre duas funções delta a equação (26) é imediatamente integrada, fornecendo

$$
\theta_{n}=\theta_{n}^{*}+\omega_{0} T \text {. }
$$

Substituindo a equação 29 na equação $(30)$ e trocando $n$ por $n+1$, obtemos

$$
\theta_{n+1}=\theta_{n}+2 \pi \frac{\omega_{0}}{\omega}+\varepsilon g\left(\theta_{n}\right) \equiv F\left(\theta_{n}\right) .
$$

Na dinâmica não-linear uma relação deste tipo é chamada uma equação a diferenças, mapeamento, 
ou simplesmente mapa. Partindo de uma condição inicial $\theta_{0}$ nós obtemos os valores em tempos posteriores iterando a relação acima:

$$
\theta_{1}=F\left(\theta_{0}\right), \quad \theta_{2}=F\left(\theta_{1}\right)=F\left(F\left(\theta_{0}\right)\right), \quad \text { etc. }
$$

o que numericamente é muito mais rápido do que a integração de um sistema de equações diferenciais como (3)-(4). Não é nosso objetivo, neste trabalho, entrar em detalhes sobre a dinâmica de mapas, porém o leitor interessado poderá encontrar mais informações a respeito nos livros-texto, como 16 e 23$]$.

Como $\theta \in[0,2 \pi)$ a relação (31) é denominada um mapa do círculo. Podemos redefinir as variáveis como

$$
\phi \equiv \frac{\theta}{2 \pi}, \quad \nu \equiv \frac{\omega_{0}}{\omega},
$$

de forma que o mapa (31) é definido no intervalo $[0,1)$ :

$$
\phi_{n+1}=\phi_{n}+\nu+\frac{\varepsilon}{2 \pi} g\left(2 \pi \phi_{n}\right), \quad(\bmod 1)(33)
$$

onde a prescrição mod 1 indica que devemos reduzir o valor de $\phi$, sempre que necessário, ao domínio $[0,1)$. A escolha $g(x)=-\sin (x)$ leva-nos ao chamado mapa do seno-círculo, bastante estudado na literatura da dinâmica não-linear 22, 24]:

$$
\phi_{n+1}=\phi_{n}+\nu-\frac{\varepsilon}{2 \pi} \sin \left(2 \pi \phi_{n}\right) .
$$

Para um mapa, no qual o tempo é uma variável discreta, o papel da frequência é desempenhado pelo chamado número de rotação 24

$$
\Omega=\lim _{n \rightarrow \infty} \frac{\phi_{n}-\phi_{0}}{n}
$$

Na figura 8 mostramos o número de rotação em função da intensidade do forçamento $\varepsilon$ e da razão entre as frequências $\nu$ para o mapa (34). Podemos observar a existência de uma grande região de sincronização ancorada em $\nu=0 \operatorname{com} \Omega=0$. Devido à periodicidade do intervalo $[0,1)$ esta região corresponde à metade da língua de Arnold correspondente à ressonância 1 : 1. A outra metade, $\operatorname{com} \Omega=1$,

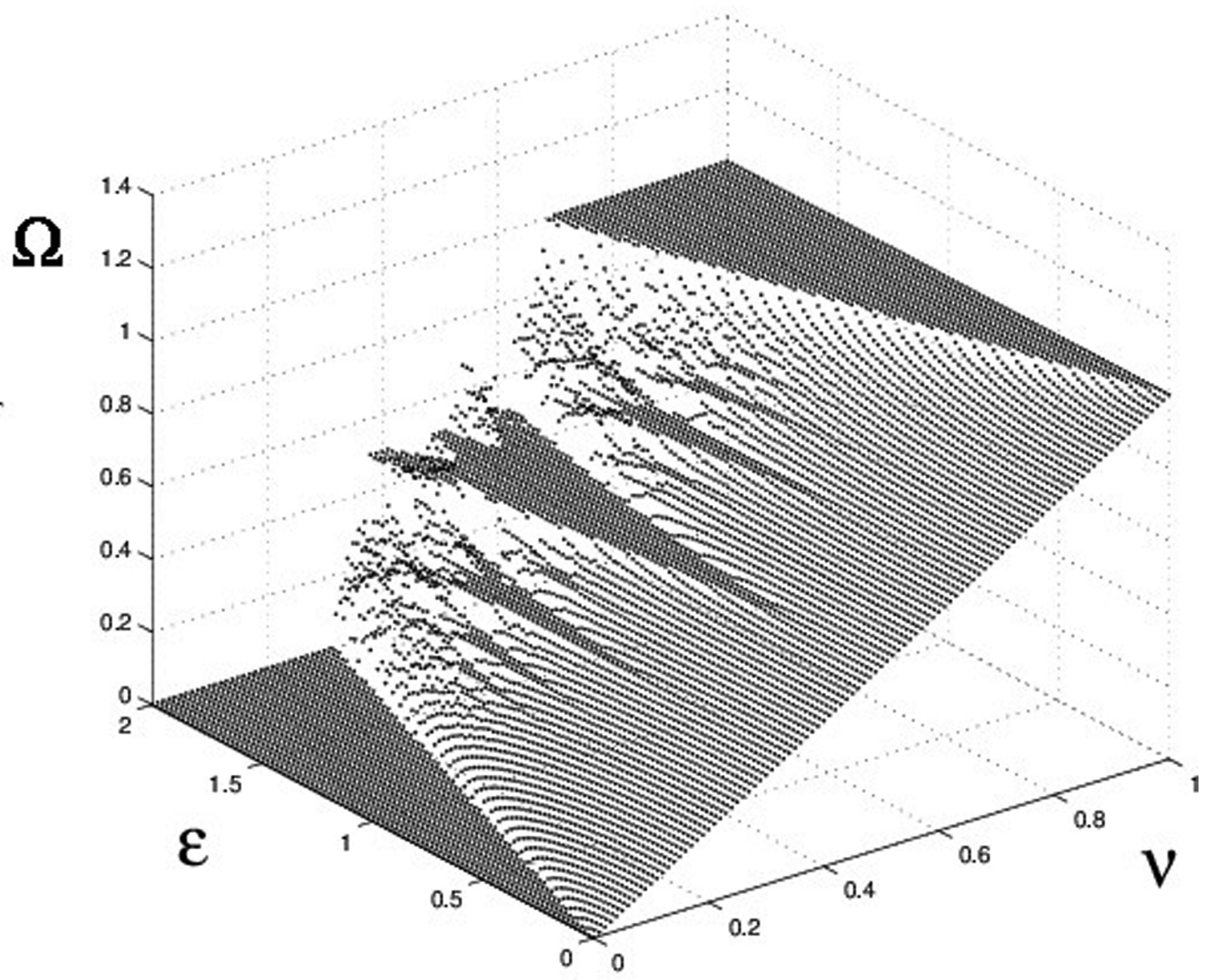

Figura 8: Número de rotação do mapa do seno-círculo como função dos parâmetros $\varepsilon$ e $\nu$. 
está ancorada em $\nu=1$. Uma língua de Arnold bem menor está ancorada em $\nu=1 / 2$, e corresponde à região de sincronização generalizada $1: 2$ ou $\omega=2 \omega_{0}$. Podemos, ainda, divisar outras línguas de Arnold mais estreitas, ancoradas em pontos intermediários.

Na verdade, para $\varepsilon$ muito pequeno, é até difícil divisar os pontos onde estas línguas são ancoradas no eixo dos $\nu$, mas sabemos pela nossa discussão anterior (baseada na hipótese de forçamento débil) que haverá línguas (ainda que muito estreitas) ancoradas em todos os valores racionais $m / n$ ( $m$ e $n$ primos entre si) dentro do intervalo $\nu \in[0,1]$. O conjunto de números racionais é denso no intervalo $[0,1]$, ou seja, entre dois racionais sempre haverá um terceiro racional 25 . Em particular, é possível mostrar que (propriedade de Farey) 26]:

$$
\frac{m}{n}<\frac{m+m^{\prime}}{n+n^{\prime}}<\frac{m^{\prime}}{n^{\prime}} .
$$

Por exemplo, entre as línguas $0 / 1$ e $1 / 1$ existe a língua $1 / 2$. Entre esta e as primeiras há línguas $1 / 3$ e $2 / 3$, e assim por diante.

$\mathrm{Na}$ aproximação de forçamento débil nós mostramos que as regiões de estabilidade são retangulares mas, à medida em que $\varepsilon$ cresce, a figura 8 sugere que as fronteiras das línguas de Arnold correspondentes tornam-se curvilíneas. Para forçamento ainda mais intenso vemos que as línguas tornam-se ainda mais complicadas, podendo bifurcar-se, e vários comportamentos dinâmicos aparecem, como caos e multiestabilidade [22,24.

Na seção anterior vimos que o comportamento do oscilador fora das línguas de Arnold é irregular, ou quase-periódico. Usando o mapa do seno-círculo (34) é fácil mostrar que, enquanto dentro de uma região de sincronização o valor do número de rotação é racional, fora desta região, o número de rotação é irracional.

No diagrama da figura 8 cada região de sincronização está associada a um platô do número de rotação, de forma análoga ao que vimos no caso contínuo (vide Fig. 6). Para $\varepsilon \leq 1$ esta sequência de platôs é chamada de "escada do diabo", pois entre cada dois degraus há sempre um terceiro (devido à propriedade de Farey já mencionada), de modo que resulta numa escada impossível de subir [27]. Quando $\varepsilon<1$ esta escada é incompleta, no sentido de que entre dois platôs há intervalos onde o número de rotação é irracional. Para $\varepsilon=1$ pode-se mostrar que esta escada é completa, ou seja, todos os degraus são racionais 27

Na figura 9(a) nós mostramos um gráfico do número de rotação versus $\nu$ para o caso $\varepsilon=1$. A escada do diabo é auto-similar, que é uma característica de fractalidade: ampliando partes da escada obtemos figuras similares a ela em diferentes escalas [Figs. 9(b) e (c)]. Como as larguras das regiões de sincronização aumentam $\operatorname{com} \varepsilon$, quando $\varepsilon>1$ ocorrem superposições das línguas de Arnold, levando a multi-estabilidade e caos, fenômenos bastante complexos que não serão discutidos neste artigo.

\section{Conclusões}

Neste trabalho abordamos matematicamente o forçamento externo periódico de osciladores auto-sustentados. Basicamente temos um acoplamento (nãolinear e unidirecional) entre dois osciladores de fase. O tratamento que demos é bastante geral, no sentido de que a função que implementa o forçamento
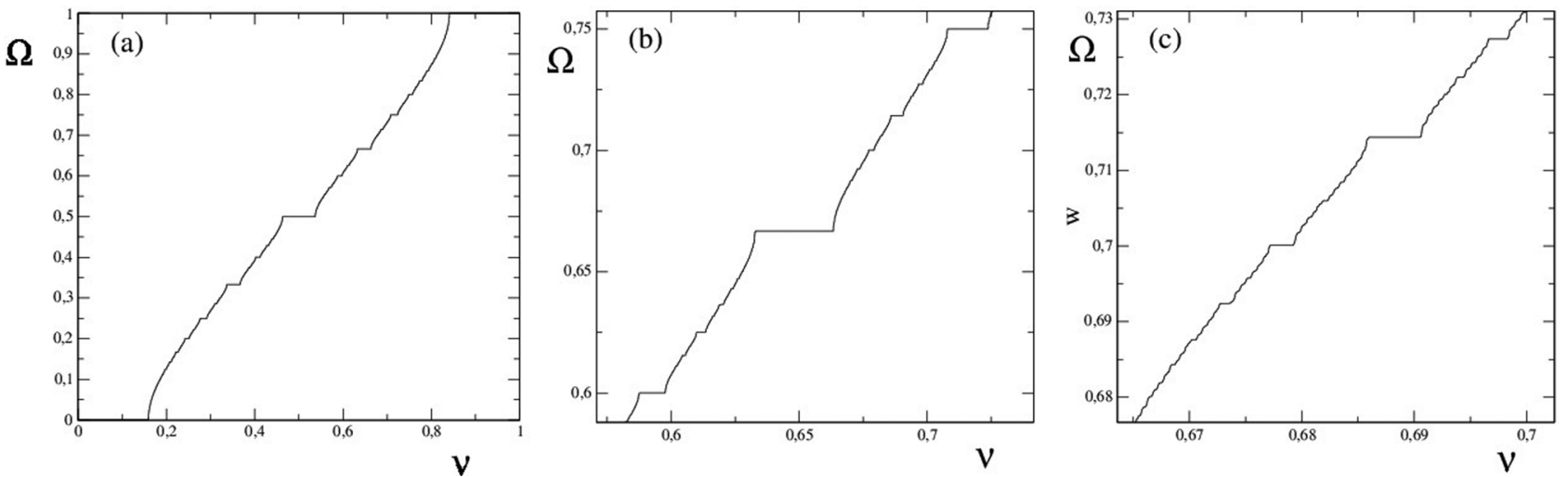

Figura 9: (a) Número de rotação do mapa do seno-círculo como função de $\nu$, para $\varepsilon=1$. (b) e (c) são ampliações de partes da figura (a). 
pode ser arbitrária, com a única restrição de ser periódica nas fases. Para tornar a discussão mais concreta, estudamos com mais detalhe o caso onde o forçamento é senoidal, redundando na chamada equação de Adler, bastante usada na eletrônica.

No caso em que a frequência do forçamento seja próxima à frequência natural do oscilador de fase mostramos que o oscilador ajusta sua frequência à do forçamento, desde que a diferença entre as frequências seja suficientemente pequena. Mais precisamente, desde que esta diferença, ou descasamento, seja no máximo igual à intensidade do forçamento, que é suposta pequena o suficiente para o uso de uma aproximação que destaca apenas os termos ressonantes. Além da sincronização entre as frequências ("entrainment"), há um travamento de fase: a diferença entre as fases do oscilador e do forçamento é constante no tempo.

Se a frequência do forçamento é muito diferente da frequência do oscilador (grande descasamento) não há mais sincronização de frequências, e o oscilador passa a ter uma fase cuja evolução temporal não é mais uniforme. Dizemos que o movimento, nesse caso, é quase-periódico, e caracterizado por uma frequência de batimento que cresce com a intensidade do forçamento. Na vizinhança da transição entre sincronização e batimento este crescimento obedece uma lei de escala do tipo raiz quadrada.

Podemos ter, ainda, uma ressonância generalizada, na qual as frequências natural e do forçamento são comensuráveis, ou seja, sua razão é um número racional (o quociente entre dois números inteiros co-primos $m$ e $n$ ). Na vizinhança desta ressonância generalizada há também uma sincronização entre frequências e um travamento de fase. As regiões de sincronização $m: n$, chamadas línguas de Arnold, têm formato triangular, tamanhos diferentes e usualmente menores do que a ressonância $1: 1$.

O caso em que a intensidade do forçamento é grande não permite um tratamento geral, demandando alguma forma de solução do sistema de equações que descreve os dois osciladores acoplados. Isto pode ser feito no caso particular em que o forçamento consiste de pulsos do tipo função delta aplicados em intervalos regulares de tempo, o que permite a construção de um mapa, ou equação a diferenças.

A análise dinâmica deste mapa mostra que as línguas de Arnold tornam-se mais alargadas e deixam de ter um formato triangular quando o forçamento é grande. De forma geral, os platôs de sin- cronização de frequência são separados por regiões de quase-periodicidade e formam uma "escada do diabo". Já para forçamento extremamente alto a análise dinâmica é mais complicada, podendo levar a fenômenos como multiestabilidade e caos, não abordados neste trabalho. Além disto, é importante considerar também o caso em que o acoplamento entre os dois osciladores é bidirecional, ou seja, ambos são influenciados pelo acoplamento. Esta análise, que em muitos aspectos espelha a que fizemos aqui, será deixada para um próximo trabalho.

\section{Agradecimentos}

Este trabalho teve o apoio financeiro do $\mathrm{CNPq}$ e da CAPES.

\section{A. Termos ressonantes e médias temporais}

Na equação (3), a função $G(\theta, \psi)$, sendo periódica em ambas as variáveis angulares, pode ser desenvolvida em série de Fourier dupla, na forma complexa

$$
\begin{aligned}
G(\theta, \psi) & =\sum_{k=-\infty}^{\infty} \sum_{\ell=-\infty}^{\infty} G_{k \ell} e^{i(k \theta+\ell \psi)} \\
& =\sum_{k, \ell} G_{k \ell} e^{i(k \theta+\ell \omega t)}
\end{aligned}
$$

onde $G_{k \ell}$ são coeficientes de Fourier. Na ausência do forçamento externo $(\varepsilon=0)$ a equação (4) tem a solução $\theta(t)=\omega_{0} t$, a qual pode ser usada na equação (36) como aproximação de ordem mais baixa:

$$
G(\theta, \psi)=\sum_{k, \ell} G_{k \ell} e^{i\left(k \omega_{0}+\ell \omega\right) t} .
$$

Próximos à ressonância $\left(\omega \approx \omega_{0}\right)$ temos que os termos com $\ell=-k$ na equação (37), na forma $e^{i k\left(\omega_{0}-\omega\right) t}$, oscilam lentamente com o tempo, ao passo que todos os demais têm variações comparativamente rápidas:

$$
G(\theta, \psi)=\sum_{\ell} G_{-\ell, \ell} e^{i \ell\left(\omega-\omega_{0}\right) t}+\sum_{k \neq-\ell, \ell} G_{k \ell} e^{i\left(k \omega_{0}+\ell \omega\right) t} .
$$

Substituindo a equação (38) na equação (3) vemos que os termos que oscilam rapidamente com o tempo são da ordem de $\varepsilon$, ou seja, têm contribuições pequenas à dinâmica do oscilador de fase. Por outro lado, os termos ressonantes do forçamento (ou seja, 
aqueles que oscilam lentamente) são os mais relevantes na resposta do oscilador. Dessa forma, podemos efetuar uma média temporal da função $G(\theta, \psi)$ sobre os termos que oscilam rapidamente, deixando apenas os termos ressonantes, o que capturará os aspectos essenciais do problema [5].

Sendo $T=2 \pi / \omega$ o período do forçamento então a média temporal é definida como

$$
\langle G(\theta, \psi)\rangle=\frac{\omega}{2 \pi} \int_{0}^{2 \pi / \omega} G(\theta, \psi) d t
$$

de modo que

$$
\begin{aligned}
\langle G(\theta, \psi)\rangle & =\sum_{\ell} G_{-\ell, \ell} e^{i \ell\left(\omega-\omega_{0}\right) t} \\
=\sum_{\ell} G_{-\ell, \ell} e^{-i \ell(\theta-\psi)} & \equiv g(\theta-\psi)=g(\varphi) .
\end{aligned}
$$

O caso de ressonância generalizada $\left(n \omega \approx m \omega_{0}\right)$ pode ser tratado de forma análoga. Separando o termo ressonante dos demais teremos

$$
\begin{aligned}
G(\theta, \psi) & =\sum_{k=-m j, \ell=n j} G_{-m j, n j} e^{i j\left(n \omega-m \omega_{0}\right) t} \\
& +\sum_{k \neq-m j, \ell \neq n j} G_{k \ell} e^{i\left(k \omega_{0}+\ell \omega\right) t} .
\end{aligned}
$$

Sendo $\Delta \omega \approx 0$ e fazendo uma média sobre o tempo nós retemos apenas os termos ressonantes, a saber:

$$
\langle G(\theta, \psi)\rangle=\sum_{j} G_{-m j, n j} e^{i j(n \psi-m \theta)} \equiv \hat{g}(\varphi) .
$$

Quando desenvolvemos em série de Fourier a função $G(\theta, \psi)$, como na equação (36), é comum que os coeficientes de Fourier dependam dos inteiros na forma $G_{k \ell} \sim k^{-a} \ell^{-b}$, onde $a$ e $b$ são expoentes positivos. Então, para cada par $k=m, \ell=n$ os coeficientes são menores do que os da ressonância principal por fatores do tipo $1 /\left(m^{a} n^{b}\right)$. Assim, para um mesmo valor da intensidade do forçamento, o tamanho dos intervalos de forçamento é menor do que o da ressonância principal pelos mesmos fatores.

\section{Referências}

[1] H.M. Nussenzveig, Curso de Física Básica. Vol. 3: Eletromagnetismo (Ed. Edgard Blücher, São Paulo, 2003).

[2] A. Chaves, Física Básica: Eletromagnetismo (LTC, Rio de Janeiro, 2007).

[3] R.L. Viana, Rev. Bras. Ens. Fis. 33, 3304 (2011); 33, 3305 (2011).
[4] P. Hagedorn, Oscilações Não-Lineares (Ed. Edgard Blücher, São Paulo, 1984).

[5] A. Pikowsky, M. Rosenblum and J. Kurths, Synchronization: A Universal Concept in Nonlinear Sciences (Cambridge University Press, New York, 2001).

[6] K. Popp and P. Stelter, Proc. R. Soc. London A 332, 1624 (1990).

[7] M. Bennett, M.F. Schatz, H. Rockwood and K. Wiesenfeld, Proc. R. Soc. London A 458, 2019 (2002).

[8] M. Headrick, IEEE Contr. Syst. 22, 41 (2002).

[9] J. Pantaleone, Am. J. Phys. 70, 992 (2002).

[10] G.F. Herrenden-Harker, Am. J. Phys. 8, 1 (1940).

[11] M.F. Bear, B.W. Connors and M.A. Paradiso Neuroscience: Exploring the Brain (Lippincott, Philadelphia, 2006), 3rd ed.

[12] E.R. Kandel, J.H. Schwartz and T.M. Jessell, Principles of Neural Science (McGraw-Hill, New York, 2012), 5th ed.

[13] L. Squire, D. Berg, F. E. Bloom, S. du Lac, A. Ghosh and N. C. Spitzer (Editors), Fundamental Neuroscience, 4th Ed. (Academic Press, New York, 2012).

[14] C.A.S. Batista, R.L. Viana, S.R. Lopes and A.M. Batista, Physica A 410, 628 (2014).

[15] J.L. Hindmarsh and R.M. Rose, Proc. R. Soc. London B 221, 87 (1984).

[16] L.H.A. Monteiro, Sistemas Dinâmicos (Editora da Física, São Paulo, 2007), $2^{\mathrm{a}}$ ed.

[17] R. Adler, A Study of Locking Phenomena in Oscillators, Proceedings of the IRE 34, 351 (1946).

[18] R.V. dos Santos and L.H.A. Monteiro, Comm. Nonlin. Sci. Numer. Simulat. 17, 2722 (2012).

[19] C. Johnson, Chronobiology: Biological Timekeeping (Sunderland, Massachusetts, 2004).

[20] N. Marques e L. Menna-Barreto (organizadores), Cronobiologia: Princípios e Aplicações (Editora da Universidade de Buenos Aires/Edusp/Fiocruz, São Paulo, 1997).

[21] S.H. Strogatz, Nonlinear Dynamics And Chaos: With Applications To Physics, Biology, Chemistry, and Engineering (Westview Press, Boulder, 2001).

[22] E. Ott, Chaos in Dynamical Systems (Cambridge University Press, Cambridge, 1997).

[23] N. Fiedler-Ferrara e C.P.C. do Prado, Caos: Uma Introdução (Ed. Edgard Blücher, São Paulo, 1994).

[24] M.H. Jensen, P. Bak and T. Bohr, Phys. Rev. A 30, 1960 (1984).

[25] E.L. Lima, Análise Real: Funções de Uma Variável, Vol. 1 (Coleção Matemática Universitária, IMPA, Rio de Janeiro, 2016), 12 a ed.

[26] S.B. Guthery, A Motif of Mathematics: History and Application of the Mediant and the Farey Sequence (Docent Press, Boston, 2011).

[27] P. Bak, Physics Today 39, 38 (1986). 